\title{
Analysis of a Multi-Access Scheme and Asynchronous Transmit- Only UWB for Wireless Body Area Networks
}

\author{
Ho Chee Keong, Student Member, IEEE, and Mehmet R. Yuce, Member, IEEE
}

\begin{abstract}
Ultra Wideband (UWB) has many favorable factors for use in a wireless body area network application. The major drawback is the high power consumption of an UWB receiver. One solution to address this problem is to use a transmit-only UWB sensor node. In this paper, we propose a multi-access scheme that is suitable for asynchronous transmitonly UWB wireless body area networks (UWB-WBAN). Each sensor attached on the patient under monitoring is assigned a unique number of UWB pulses per data bit. The number of UWB pulses assigned to the sensors is optimized to improve the bit error rate and system reliability. Simulation shows that through careful selection of the number of pulses for the sensors, it is possible to maintain almost similar bit error probability, regardless of the distance from the receiver.
\end{abstract}

\section{INTRODUCTION}

$\mathrm{T}$ HE advance in technologies have improved the quality and efficiency of health care services. Wireless Body Area Network (WBAN) is one of such emerging technologies that find application in healthcare systems. Deployment of WBAN in an elderly home will improve the medical staff efficiency and provide the elderly with more freedom in movement. The use of WBAN from home or in hospital, to transmit real time information, allows for timely medical treatment in crucial situations. With the aid of WBAN, patients suffering from illness that requires long term monitoring can lead a less disrupted life and reduces the frequency of visiting a medical staff. There is no doubt in the growing importance of WBAN, especially in the current situation, where aging population and scarce medical resources is a problem faced in many developed countries. Therefore in November 2007, a task group (IEEE802.15.6) was formed to develop a standard for WBAN.

WBAN is a special purpose wireless sensor network consisting of several sensor nodes that sense biological information from human body. The data from sensors are transmitted over a short distance wirelessly to a control device worn on the body or placed at an accessible location. The collected data from the control devices are then transferred to remote destinations in a wireless body area network. The key requirements of the sensor nodes for WBAN are extremely low power consumption, low complexity MAC (medium access control) protocol, scalable data rate (up to $10 \mathrm{Mbps}$ ) and small form factor [1]. Several

This work was supported in part by the Australian Research Council (ARC) under Discovery Projects Grant.

Ho Chee Keong and M. R. Yuce are with the School of Electrical Engineering and Computer Science, University of Newcastle, Callaghan, NSW 2308, Australia (e-mail: mehmet.yuce@newcastle.edu.au ).
Table 1: Comparison of sensor node wireless platforms

\begin{tabular}{|c|c|c|c|c|c|}
\hline \multirow[t]{2}{*}{ Model } & \multirow{2}{*}{$\begin{array}{l}\text { Com- } \\
\text { pany }\end{array}$} & \multirow[t]{2}{*}{ Freq } & \multirow{2}{*}{$\begin{array}{l}\text { Data } \\
\text { Rate }\end{array}$} & \multicolumn{2}{|c|}{ Current } \\
\hline & & & & $\mathbf{T x}$ & $\mathbf{R x}$ \\
\hline UWB & $\begin{array}{c}\text { Our } \\
\text { design }\end{array}$ & $\begin{array}{c}3.1-10.6 \\
\mathrm{GHz}\end{array}$ & $\begin{array}{c}10 \\
\text { Mbps }\end{array}$ & $\begin{array}{r}15 \\
\mathrm{~mA}\end{array}$ & - \\
\hline $\begin{array}{l}\text { Mica2 } \\
\text { (MPR400) }\end{array}$ & $\begin{array}{c}\text { Cross- } \\
\text { bow }\end{array}$ & $\begin{array}{c}868 / 916 \\
\mathrm{MHz}\end{array}$ & $\begin{array}{l}38.4 \\
\text { kbps }\end{array}$ & $\begin{array}{r}27 \\
\mathrm{~mA}\end{array}$ & $\begin{array}{r}10 \\
\mathrm{~mA}\end{array}$ \\
\hline MicAz & $\begin{array}{c}\text { Cross- } \\
\text { bow }\end{array}$ & $2.4 \mathrm{GHz}$ & $\begin{array}{c}250 \\
\text { kbps }\end{array}$ & $\begin{array}{l}17.4 \\
\mathrm{~mA}\end{array}$ & $\begin{array}{l}19.7 \\
\mathrm{~mA}\end{array}$ \\
\hline $\begin{array}{l}\text { Mica2- } \\
\text { DOT }\end{array}$ & $\begin{array}{c}\text { Cross- } \\
\text { bow }\end{array}$ & $433 \mathrm{MHz}$ & $\begin{array}{l}38.4 \\
\text { kbps }\end{array}$ & $\begin{array}{r}25 \\
\mathrm{~mA}\end{array}$ & $\begin{array}{c}8 \\
\mathrm{~mA}\end{array}$ \\
\hline CC1010 & TI & $\begin{array}{c}300 \text { to } \\
1000 \mathrm{MHz}\end{array}$ & $\begin{array}{c}65 \\
\text { kbps }\end{array}$ & $\begin{array}{c}26.6 \\
\mathrm{~mA}\end{array}$ & $\begin{array}{l}11.9 \\
\mathrm{~mA}\end{array}$ \\
\hline CC2400 & TI & $2.4 \mathrm{GHz}$ & $\begin{array}{c}1 \\
\text { Mbps }\end{array}$ & $\begin{array}{r}19 \\
\mathrm{~mA}\end{array}$ & $\begin{array}{r}23 \\
\mathrm{~mA} \\
\end{array}$ \\
\hline
\end{tabular}

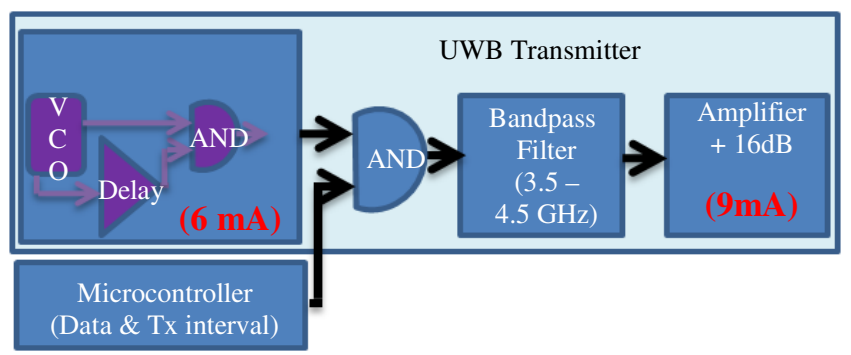

Figure 1: UWB transmitter block diagram

propriety solutions based on Bluetooth, ZigBee, WLAN and ISM band are available, but they are not optimised for the WBAN applications. Some of the commonly used wireless sensor node platforms from Crossbow [2] and Texas Instrument [3] are shown in Table 1. An Ultra Wideband (UWB) transmitter assembled using off-shelves components is also included in Table 1 for comparison. Block diagram of the impulse based UWB transmitter is shown in Figure 1. The narrow UWB pulse is generated by the narrow pulse generator and the role of the amplifier is to increase the bit energy of the pulse to as close to the UWB limit as possible. From Table 1, the power consumed per data bit for a UWB transmitter is much lower as compared to other narrowband wireless technologies. Based on the key requirements for sensor nodes, UWB seems to be the best choice in term of power consumption, scalability and size. But, one major drawback for UWB wireless scheme is that the UWB receiver circuit consumes much more power than the transmitter. One method to overcome this problem is to use a transmit-only (Tx-only) UWB sensor node.

Tx-only sensor nodes have been analyzed by [4, 5], in which they focused mainly on maximizing data throughput 
by means of data admission policies on the receiver end. A major challenge faced in a Tx-only system is collision avoidance, which is not covered in $[4,5]$. Due to the asynchronous and uncoordinated transmission nature of a Tx-only sensor node, there is a high probability of collision in a multiuser environment. The collision can be individual or clustered. A high collision rate will have harmful effect on the reliability of the system, which is an important criterion to be considered for sensor nodes carrying vital physiological signals. Therefore it is important to minimize the chances of collision and thus loss of data due to collisions. In [6], a multi-access scheme for asynchronous users, where each user is identified with a unique pulse rate is analysed in detail. The scheme requires a dedicated receiver for each sensor node. In a WBAN scenario, there is only one receiver per user, receiving data from multiple sensor nodes. The scheme proposed by [6], can be used to identify individual users in a WBAN environment, but not for differentiating the various sensor nodes for each user. As it would greatly increase the complexity and reliability of the receiver, due to need for synchronizing the different pulse rate using one receiver. The bit error rate (BER) for the scheme proposed in [6] is optimized solely based on the collision probability. The BER due to low average transmission power are not taken into consideration, which is very applicable in a wireless body sensor scenario.

In this paper, we propose a Tx-only multi access scheme that is catered for WBAN applications. The scheme has been optimized by taken into consideration the BER due to both collision and low signal to noise ratio $(S N R)$. The proposed scheme identifies the individual sensor node by the number of pulses in a bit and the users by different pulse rates.

\section{TRANSMIT POWER CONSIDERATION FOR SENSOR NODES}

Table 2 shows a list of the commonly monitored physiological signals in a WBAN. In general, most physiological signals have low sampling rate of less than 1 sample per milliseconds. Each sample is approximately between 8 to 12 bits, depending on the resolution of the analog to digital converter used. Figure 2 shows the transmission pattern of a typical physiological signal when a much higher data rate is used to output the sampled information. The transmission pattern resembles that of a gating signal, where the interval between each transmission $\left(T_{i n t}\right)$ is much longer than the transmission slot $\left(T_{\text {slot }}\right)$. In 2005, FCC allows the peak and average measurement of a gated signals to be measured with gating on [7]. The relationship between the peak and average power of a gated signal can be represented by (1).

$$
\begin{aligned}
& P_{\text {peak }}=P_{\text {ave }}+10 \log \left(\frac{B_{\text {peak }}}{B_{\text {ave }}}\right)+P_{P A P R, \text { slot }}+10 \log \left(\frac{T_{\text {int }}}{T_{\text {slot }}}\right) \\
& \text { where, } P_{P A P R, \text { slot }}=P A P R_{P S}+10 \log \left(\frac{T_{P}}{T_{C}}\right)
\end{aligned}
$$

Table 2: List of common physiological signals

\begin{tabular}{|l|c|c|}
\hline Vital Signs & $\begin{array}{c}\text { Monitoring } \\
\text { Period }\end{array}$ & $\begin{array}{c}\text { Sampling } \\
\text { rate }\end{array}$ \\
\hline $\begin{array}{l}\text { Electrocardiogram } \\
(\text { ECG })\end{array}$ & Continuous & 300 samples/s \\
\hline $\begin{array}{l}\text { Electroencephalogram } \\
(\text { EEG })\end{array}$ & Continuous & 200 samples/s \\
\hline Electrooculogram (EOG) & Continuous & 200 samples/s \\
\hline Heart beat rate & Every 1 sec & \\
\hline $\begin{array}{l}\text { Oxygen Saturation } \\
\text { (SP02) }\end{array}$ & Every 1 sec & \\
\hline Blood pressure & Every 1 min & \\
\hline Temperature & Every 1 min & \\
\hline
\end{tabular}

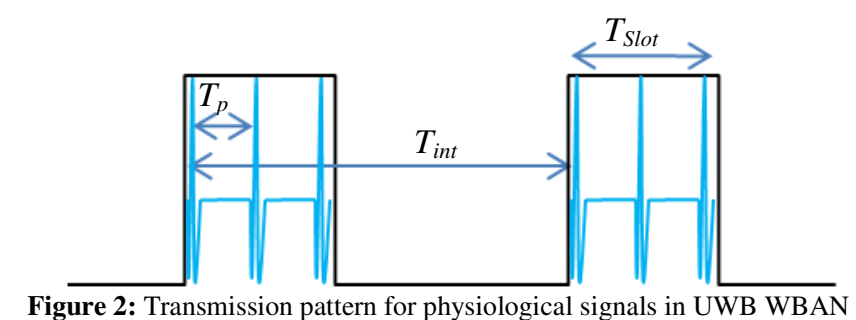

Figure 2: Transmission pattern for physiological signals in UWB WBAN

The peak power limit $\left(P_{\text {peak }}\right)$ is $0 \mathrm{dBm}$ based on a peak measurement bandwidth $\left(B_{\text {peak }}\right)$ of $50 \mathrm{MHz}$, while the average power limit $\left(P_{\text {ave }}\right)$ is $-41 \mathrm{dBm}$ based on an average measurement bandwidth $\left(B_{\text {ave }}\right)$ of $1 \mathrm{MHz} . P_{P A P R \text {, slot }}$ is the peak to average power ratio $(P A P R)$ in the transmission slot, which is inversely proportional to average power in $T_{\text {slot }}$. $P A P R_{P S}$ is the peak to average ratio of the UWB pulse, which is dependent on the pulse shape. $T_{p}$ is the interval between pulses and $T_{c}$ is the pulse width.

From (1), a low duty cycle gating system with $T_{\text {int }}$ larger than $1 \mathrm{~ms}$ is peak power limited. As $P_{\text {ave }}$ is much lower than $-41 \mathrm{dBm}$, there is much room for sending more pulses of equal bit energy. In a low duty cycle gating system, it is possible to improve the system performance by increasing the average power in $T_{\text {slot }}$ to more than $-41 \mathrm{dBm}$, while meeting the UWB regulation. Average power in $T_{\text {slot }}$ can be increased by either decreasing the pulse interval $\left(T_{p}\right)$ or sending more pulses per bit. The relationship between the number of pulses per bit $\left(N_{S}\right)$ and the $S N R$, without multipleaccess interference $(M A I)$ can be represented by (2). $E_{b}{ }^{(i)}$ is the energy of $i^{\text {th }}$ user and $n_{0}$ is the white Gaussian noise. In the presence of $M A I$, the signal to inference and noise ratio (SINR) of the system is given by (3) [8]. From (2) and (3), decreasing the pulse interval, while keeping the transmission slot constant, improves the SNR but not the SINR. Both SNR and $S I N R$ can be improved by sending more pulses, while keeping pulse interval constant. In our proposed multiaccess scheme, the latter method is employed to improve the system $S N R$ and SINR. Figure 3 shows the plot of bit error rate $(B E R)$ against distance. As distance increases, $B E R$ increases. Transmitting more pulses per bit helps to maintain the same $B E R$ as the distance increases. 


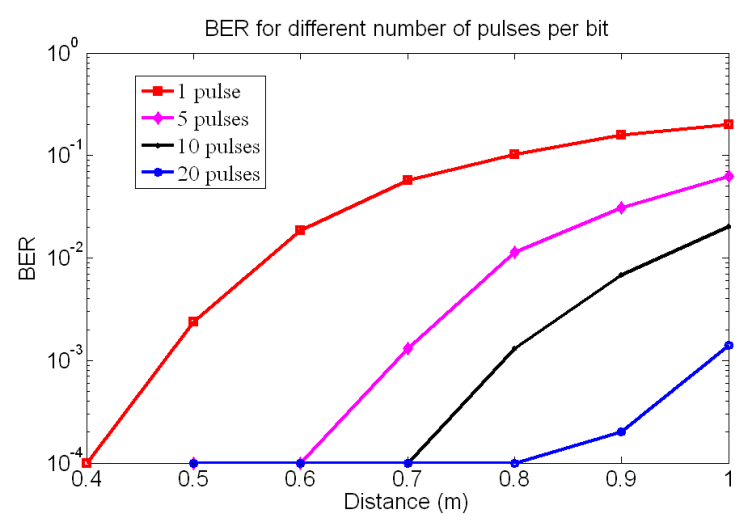

Figure 3: BER for different number of pulses per bit.

$$
\begin{gathered}
\operatorname{SNR}^{(i)}=\frac{N_{S} E_{b}{ }^{(i)}}{n_{0}} \\
\operatorname{SINR}^{(i)} \approx \frac{N_{S} E_{b}^{(i)}}{n_{0}+\frac{1}{n_{c}} \sum_{k=1}^{N_{u}} E_{b}{ }^{(k)}}, n_{c}=\frac{T_{P}}{T_{C}}
\end{gathered}
$$

\section{TX-ONLY MULTI-ACCESS SCHEME FOR WBAN}

Consider a multiuser scenario with $M$ patients under monitoring, the patients/users are index with number $m €$ $\{1,2, \ldots, M\}$. Assume each user has $K$ sensors attached to it, the sensors are index with number $k \in\{1,2, \ldots, K\}$. The transmit signal of the proposed PPM (pulse position modulation) modulated asynchronous UWB Tx-only system is represented by the following model:

$$
S_{T X}^{(m, k)}(t)=\sqrt{E_{b}} \sum_{i=0}^{\infty} \sum_{j=0}^{N_{b i t}-1} \sum_{n=0}^{N_{s}-1} W_{t x}\left(\begin{array}{l}
t-i T_{\mathrm{int}}^{(k)}-j T_{b}^{(k)} \\
-n T_{p}^{(m)}-\Delta_{j} \tau_{m}-\tau^{(k)}
\end{array}\right)
$$

where $W t x(t)$ is the transmitted UWB pulse, $E_{b}$ is the bit energy, $T_{i n t}$ is the time interval between successive transmissions for sensor $k, T_{b}$ is the bit period, which is equal to $N_{s} T_{p}, N_{s}$ is the number of pulses per bit, $T_{p}$ is the pulse interval for user $m, \Delta_{j} \epsilon\{0,1\}$ is the information of the bit, $\tau_{\mathrm{m}}$ is the time shift for PPM modulation and $\tau^{(\mathrm{k})}$ is the asynchronous random start time of sensor $k$.

As discussed in the previous section, due to low average power of a low duty cycle gating system, there is much room for sending multiple pulses without reducing the bit energy, therefore $E_{b}$ for all users are the same for this model. $T_{i n t}$ is chosen based on [6], such that the collision probability can be minimized. In this model, each individual user is identified by a different $T_{p}$, while the sensors on each user are identified by different number of pulses per bit $\left(N_{s}\right)$.

The bit error probability (BEP) of sensor $k$ of user $m$ of the proposed system is given in (5), where $Q\left(S N R^{(m, k)}\right)$ is the bit error probability due to $S N R$ and $P_{C}$ is the average probability of collision. The BEP given in (5) is considered to be the worst case scenario, as all bits in a transmission packet are considered lost, if a collision occurs during the transmission period.

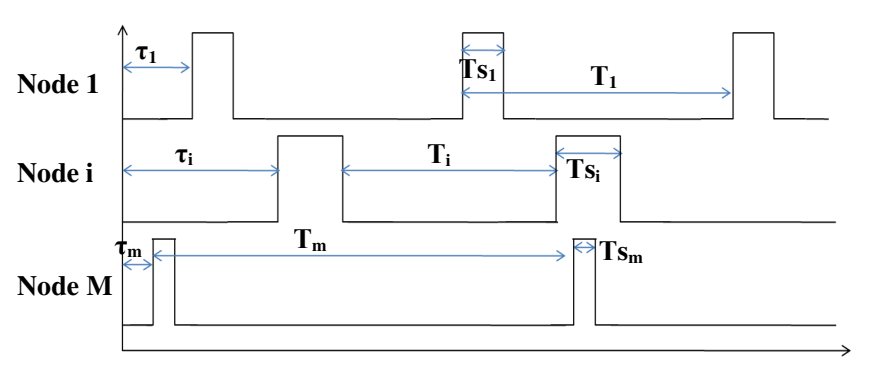

Figure 4: Transmission pattern for multi nodes

$$
B E P^{(m, k)} \approx Q\left(S N R^{(m, k)}\right)+P_{c}^{(m, k)}
$$

As shown in the simulation results depicted in Figure 3, $B E P_{S N R}$ increases as the distance between transmitter and receiver get larger. It is also noted that sending more pulses per bit helps to improve the $Q\left(S N R^{(m, k)}\right)$. But sending more pulses per bit also increases the probability of collision, therefore a balance between $Q\left(S N R^{(m, k)}\right)$ and $P_{C}$ is required to optimize the $B E P$. The key factors affecting $Q\left(S N R^{(m, k)}\right)$ are location of sensor nodes and receiver around the body in WBAN. The main factors affecting $P_{C}$ are $T_{s l o t}, T_{\text {int }}$, and the number of sensors on a user. The average collision probability for sensor $M$, as shown in (6) given by [6].

$$
P_{c}(M)=1-\prod_{j \in m \backslash\{M\}}\left(1-\frac{T_{\text {slot }}^{(j)}}{T_{\text {int }}^{(j)}}\right)
$$

Equation (6) is based on collision occurring at sampling instant of each pulse, which can also be applied to our proposed scheme, as the duty cycle for our transmitted signal is very low and we considered the whole packet to be lost if a collision has occurred. The above assumption is valid in the one pulse per bit scenario. i.e. all transmission slots are of equal length. Figure 4 shows the transmission pattern of sensor 1 , sensor $i$, and sensor $M$, where sensor $M$ is transmitting 1 pulses per bit and sensor $i$ is transmitting 3 pulses per bit. The transmission slot of sensor $i$ is 3 times that of sensor $M$. The average probability of collision given by [6], is only valid for the sensor $M$, where we consider one transmission slot as one sampling instant. It is not valid for sensor 1 and sensor $i$, as the transmission slot is equivalent to two and three sampling instants respectively. The average probability of collision of sensor $i$ is given by (7), where $n \epsilon$ $\left\{1,2, \ldots, N_{s}\right\}$ and $P_{C}(\mathrm{i}, \mathrm{j})$ is the average probability of collision of sensor $i$ at sampling instant $j$. As $P_{C}(\mathrm{i}, \mathrm{j})$ is not mutually exclusive, the peak collision probability can be given by (8).

$$
\begin{gathered}
P_{c, \text { new }}(i)=\bigcup_{j \in n} P_{c}(i, j) \\
P_{c, \text { new }}^{\text {peak }}(i)=N_{S} P_{c}(i)
\end{gathered}
$$




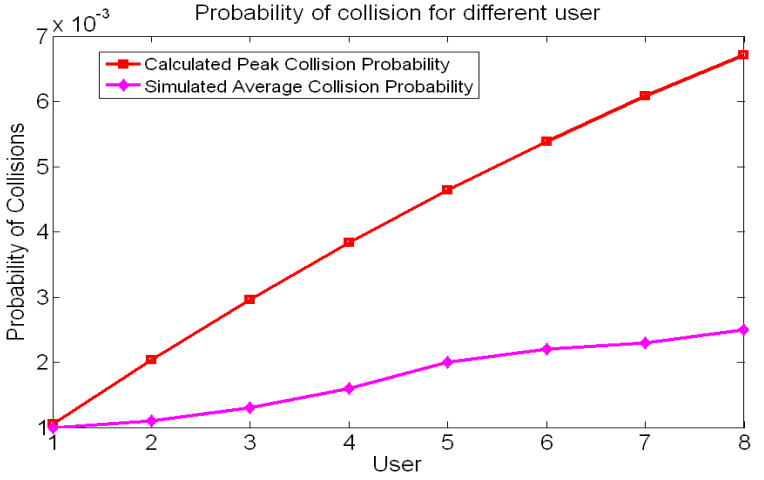

Figure 5: Probability of collision for different sensors

Figure 5 shows the peak collision probability given in (8) and the simulated average collision probability for 8 ECG sensors, each with transmission packet of 10 bits and a pulse interval of $10 \mathrm{~ns}$. The simulations are carried out with 3 sets of 10000 bits. Sensor 1 transmits 1 pulse per bit, while sensor 8 will send 8 pulses per bit and so on. Figure 5 also shows that sensor 8 has the highest probability of collision, while sensor 1 is the least. Therefore, sending more pulses per bit would result in higher collisions. From Figure 5, the peak collision probability is much higher than the simulated average probability, the differences get larger as more pulses are sent. As $P_{C}(\mathrm{i}, \mathrm{j})$ is not mutually exclusive, therefore as the number of sampling instants increases, there will be more overlapping regions, which result in larger differences between the calculated peak and simulated average collision probability. In a WBAN system, data reliability is one of the major concerns. Based on the result shown in Figure 5 it is safe to use (8) to determine the acceptable collisions allowed, when designing a WBAN system. The BEP given in (5) can be rewritten in terms of $N_{s}$ as shown in (9). Multiaccess interference is not considered, as when two sensors are transmitting at the same time, both packets are discarded. It is however accounted in the collision probability. From (9), through careful selection of $N_{s}$, it is possible to achieve the required $B E P$ for each sensor.

$$
B E P^{(m, k)} \approx Q\left(\frac{N_{s} E_{b}^{(m, k)}}{n_{0}}\right)+N_{S} P_{c}(m, k)
$$

\section{Simulation Results}

Table 3 shows the simulation specifications of a WBAN system with six sensors. Assume the required $B E P$ for all the six sensors is set as $10^{-3}$. The number of pulses per bit is selected based on (9). Each transmission slot consists of 10 data bits, and the pulse interval is set to $10 \mathrm{~ns}$. All the transmitted UWB pulses are of the same pulse shape and contain the same amount of energy. The simulated result is shown in Table 4. The result shows that it is possible to maintain almost similar $B E P$ for all sensors at different locations, by assigning a suitable $N_{S}$ to each sensor.
Table 3: A WBAN Simulation Scenario with sensor locations

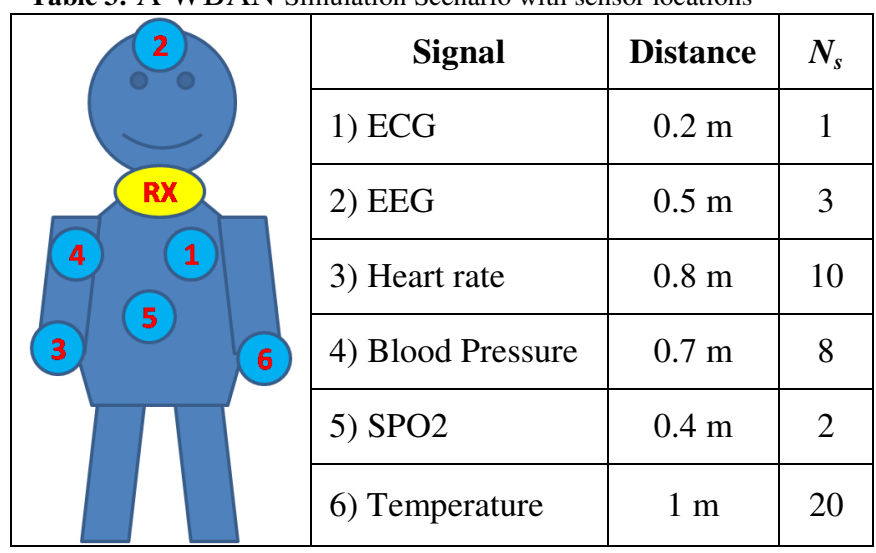

Table 4: Simulated result showing the BER changes with respect to distance and number of pulses

\begin{tabular}{|l|c|c|}
\hline \multicolumn{1}{|c|}{ Signal } & Targeted BEP & Simulated BER \\
\hline 1) ECG & 0.001 & 0.0010 \\
\hline 2) EEG & 0.001 & 0.0012 \\
\hline 3) Heart rate & 0.001 & 0.0025 \\
\hline 4) Blood Press. & 0.001 & 0.0050 \\
\hline 5) SPO2 & 0.001 & 0.0010 \\
\hline 6) Temperature & 0.001 & 0.0043 \\
\hline
\end{tabular}

\section{CONCLUSION}

In this paper, we have proposed a flexible and low power scheme for Tx-only UWB based WBAN (UWB-WBAN) systems. Variable number of pulses can be used for the sensors on the human body to improve a WBAN system performance. The selection criteria for the number of pulses should be based on the distance between the transmitting sensors and the receiver, the number of users in the system, and the transmission pattern of the sensors. Simulation results show that overall system performance can be improved by optimizing the number of pulses per bit as well as carefully locating sensors on the human body.

\section{REFERENCES}

[1] L. Huan-Bang and R. Kohno, "Introduction of SG-BAN in IEEE 802.15," IEEE ICUWB, pp. 134-139, 2007.

[2] http://www.xbow.com.

[3] http://www.ti.com/.

[4] B. Radunovic, H. L. Truong, and M. Weisenhorn, "Receiver architectures for UWB-based transmit-only sensor networks," ICUWB 2005, pp. 379-384.

[5] B. Blaszczyszyn and B. Radunovic, "Using Transmit-Only Sensors to Reduce Deployment Cost of Wireless Sensor Networks," IEEE 27th Conference on Computer Communications, 2008, pp. 1202-1210.

[6] M. Weisenhorn and W. Hirt, "Uncoordinated rate-division multipleaccess scheme for pulsed UWB signals,", IEEE Transactions on Vehicular Technology, vol. 54, pp. 1646-1662, 2005.

[7] "FCC 05-58: Petition for Waiver of the Part 15 UWB Regulations," Filed by the Multi-band OFDM Alliance Special Interest Group, ET Docket 04-352, March 11, 2005.

[8] I. Guvenc, H. Arslan, S. Gezici, and H. Kobayashi, "Adaptation of two types of processing gains for UWB impulse radio wireless sensor networks,", IET Communications, vol. 1, pp. 1280-1288, 2007. 